\title{
Power Grounding Optimization
}

\author{
Eduardo A. Cano-Plata, Oscar J. Soto-Marín, Guillermo Jiménez-Lozano, \\ Jorge H. Estrada-Estrada \\ Departamento de Ingeniería Eléctrica, Electrónica y Computación, Universidad Nacional de Colombia, \\ Manizales, Colombia \\ Email: eacanopl@unal.edu.co
}

Received 9 June 2015; accepted 22 August 2015; published 26 August 2015

Copyright (C 2015 by authors and Scientific Research Publishing Inc.

This work is licensed under the Creative Commons Attribution International License (CC BY). http://creativecommons.org/licenses/by/4.0/

\section{(c) (1) Open Access}

\begin{abstract}
In this paper we discuss the finite element models (FEM) using electromagnetic theory-Maxwell's equations. Next we developed a new procedure for optimization with the idea to be implemented in the standard IEEE-80 (2013). We expose those ideas in the paper. ETAP program and Matlab software are used for FEM.
\end{abstract}

\section{Keywords}

Finite Element Method, Grounding Systems, Circuit Model, ETAP Model, Engineering Design

\section{Introduction}

The protective scheme design is an important aspect in design and construction of the substations. The voltage gradients are created across ground mesh and points linked to earth as references [1]-[3]. The difference in potential is kept within the limits provided by IEEE Standards Documents and should be continuously monitored for the equipment's proper functionality and people safety working in surrounding.

The followings are design parameters and control that define the construction of grounding system: ground potential rise $(G P R)$, step voltage $\left(V_{\text {step }}\right)$, touch voltage $\left(V_{\text {touch }}\right)$, ground resistance $\left(R_{g}\right)$, mesh voltage $\left(V_{\text {mesh }}\right)$ and grid current $\left(I_{g}\right)$. There are various methods available for designing of ground mesh for substation; IEEE 802013 and Finite Element Method (FEM) [4] [5] are adopted for design of ground system in research conducted as these are more reliable ones.

In the grounding system design, the optimization means to find a grounding system which is able to guarantee the integrity of the equipments and continuity of the service, especially to reduce the risk of a person in the vicinity of grounded facilities being exposed to the danger of critical electric shock [1] with the minimum cost in construction of the grounding system. A new technique that uses the dynamic programming approach is proposed for optimizing the design of grounding grids to be implemented in the standard IEEE-80 (2013).

How to cite this paper: Cano-Plata, E.A., Soto-Marín, O.J., Jiménez-Lozano, G. and Estrada-Estrada, J.H. (2015) Power Grounding Optimization. American Journal of Computational Mathematics, 5, 243-252.

http://dx.doi.org/10.4236/ajcm.2015.53021 


\section{Representation of the Continuity Equation}

The abstracted definition of the finite element is following: the triple ordained $\left\langle A_{k}, B_{k}, \Omega_{k}\right\rangle$ it can be represented by $A_{k}$ the set of degree of freedom (nodes) it is a base of a space that genera the function $B_{k}$, it is normally a constitutive relation, and the domain $\Omega_{k}$ the geometrical space.

A construction of the finite element is related to the Lagrangian method and it possible to make identifying any equilibrium configuration. In this case the so-called Updated Lagrangian formulations, plus finding the spatial configuration corresponding to an instant $t$. An instant is a given value in a temporal coordinate. The notion of time here is used in a general sense; such as a coordinate that serves to number events.

The spatial configuration corresponding to the instant $t$ is defined by ${ }^{t} J$ current densities that satisfy the Maxwell continuity equation of type [6]:

$$
\nabla \cdot J=0, \quad E={ }^{t} \sigma\left({ }^{t} J\right) \quad \forall{ }^{t} \Omega
$$

where

$$
\nabla=\left(\frac{\partial}{\partial x}, \frac{\partial}{\partial y}, \frac{\partial}{\partial z}\right)
$$

The second order tensors ${ }^{t} \sigma$ are complicated nonlinear functions of ${ }^{t} J$ and of the history of the conductivity change process. A fundamental difficulty in solving Equation (1) is that the domain over which the equations must be solved is part of the solution of the problem. In essence the relationship between the constitutive Equation (1), the space $\Omega$ and the dynamic of any point $x$ in the space that satisfies the constitutive relationship is name the node of the finite element.

The mathematical problem is completed with boundary conditions at ${ }^{t} \sigma$ and ${ }^{t} J$, and it is solved using defined magnitudes over a reference configuration (in a sense, representation of the defined magnitudes in the spatial configuration) and solving a problem similar to the one described by Equation (1) over ${ }^{0} \Omega$ (where the supra index " 0 " indicates the adopted reference configuration).

The Green's conductivity tensor and the second tensor of the Kirchhoff electric field are examples of defined magnitudes over the reference configuration to solve over ${ }^{0} \Omega$ the nonlinear problems in the medium. This methodology has migrated from the techniques in mechanics of the continuous medium (computational mechanics) [7].

Simo et al. [7]-[9] describe methods used in the calculation of differentiable manifold [10] to systematize the task of finding representations over ${ }^{0} \Omega$ of tensors defined in ${ }^{t} \Omega$ and also to systematize the formulation of mathematical problems defined over ${ }^{0} \Omega$ equivalent to Equation (1). Although Maxwell developed equations in compact form, numerical methods should be used to solve them [11].

Modern developments of the finite element method applied to nonlinear problems of solid mechanics make rigorous use of these techniques [12]-[15]. The method discussed in this paper is used in electromagnetism to measure soil resistivity.

The aim of this work is to develop a geometric view of several calculation techniques of differentiable manifold such as pull-back and push-forward [16] to simply propose them as tools for engineers working on solving nonlinear problems in Continuum Mechanics using finite elements. The changes undergone by the soil in the presence of lightning must always be taken into consideration.

\section{Constitutive Relations of Maxwell's Equations}

Engineering is based on the relationship that it can have the characteristics of the materials assuming always two conservation principles are met. The first is the principle of conservation of charge and the second the principle of conservation of energy. The approach of these principles can be differential or integral form (Eulerian and Lagrangian formulation).

Assume that you have a problem which only the variable representing the electric field is considered, we can then establish the constitutive relation:

$$
{ }^{t} \underline{\underline{J}}={ }^{t} \underline{\underline{\sigma}}\left({ }^{t} \underline{\underline{E}}\right)
$$

where ${ }^{t} \underline{\sigma}$ is a tensor function in this particular case is the conductivity function that maps the two points in 
space invertible of the symmetrical space. It is important to highlight that ${ }^{t} \underline{\sigma}$ depends on the reference configuration. In this sense and since only the electrical characteristic are studied, it is understood that the principle of equipresence is respected and that no time is considered phenomenological factors of the quantum mechanics.

For studying the objectivity of the formulation, it was considered in the spatial configuration two coordinate systems, one stationary $(x)$ and other moving $\left(X^{*}\right)$, since the current density tensor is a objective special tensor can be displayed as:

$$
{ }^{t} \underline{=}=\underline{\underline{Q}}(t) \cdot{ }^{t} J^{*} \cdot \underline{Q}^{\mathrm{T}}(t)
$$

where $\underline{Q}$ is an orthogonal tensor. Since the electric field is a objective tensor

$$
{ }_{0}^{t} \underline{\underline{E}}=\underline{\underline{Q}}(t) \cdot{ }_{0}^{t} \underline{\underline{E^{*}}}
$$

Since $\underline{Q}$ is valid for any orthogonal relationship, It will also be valid for the polar decomposition, i.e.:

$$
\underline{\underline{J}}^{\mathrm{t}}={ }^{t} \underline{\underline{\sigma}}\left({ }_{0}^{t} \underline{\underline{E^{*}}}\right)
$$

Or what it is the same ${ }^{t} \underline{\underline{\sigma}}$ presents the principle dictates that the material is not affected by the change of coordinates or reference. The material is represented by the conductivity.

\section{Conductivity}

Conductivity defines the energy dissipated in instant $t$ per unit of mass, which is associated with the dissipation of electrical current in the soil. This study mainly deals with equations of a continuous medium (1). These equations in a grounding system might be represented as:

$$
\begin{aligned}
& \nabla \cdot J=0, \quad E={ }^{t} \sigma\left({ }^{t} J\right) \quad \forall{ }^{t} \Omega \\
& E=E_{\Gamma} \quad \text { en } \Gamma, \quad g_{a} \cdot J=0 \quad \text { en } \Gamma_{\Omega}
\end{aligned}
$$

In (7), $\Omega$ is the ground, $\sigma$ is its conductivity tensor, $\Gamma_{\Omega}$ is the ground surface, $g_{a}$ is the covariant vector in generalized coordinates and $\Gamma$ is the electrodes surface. The appropriate solution is the distribution of potential or the setting of potential at an arbitrary point. The dynamics must be evaluated once the grounding system acquires $\varphi_{\Gamma}$ potential (system overvoltage) and Equation (8) is calculated p.u.

$$
J=\sigma \varphi, \quad I_{\Gamma}=\iint J \mathrm{~d} \Gamma, \quad R_{e q}=\frac{\varphi_{\Gamma}}{I_{\Gamma}}
$$

In practice, the ground is considered isotropic, thereby $\sigma$ is replaced by a scalar (St. IEEE 80) [1]-[3].

In an assumed horizontal ground surface, the Dirichlet boundary condition $\Delta \varphi=0$ is shown in $\Omega$. The conditions expressed in (7) are obtained while searching for regulation. Applying to (7) Green's identity [10]:

$$
\varphi=\frac{1}{4 \Pi \sigma} \iint_{\varepsilon \in \Gamma} k(x, \varepsilon) J(\varepsilon) \mathrm{d} \Gamma, \quad x \in \Omega
$$

With weak nucleus:

$$
k(x, \varepsilon)=\left(\frac{1}{r(x, \varepsilon)}+\frac{1}{r\left(x, \varepsilon^{\prime}\right)}\right), \quad r(x, \varepsilon)=|x-\varepsilon|
$$

The functional of the problem is expressed in (5). Discretizing gives:

$$
J(\varepsilon)=\sum_{i+1}^{N} J_{i} N_{i}(\varepsilon) \quad \Gamma=\bigcup_{\alpha=1}^{M} \Gamma^{\alpha}
$$

Matlab Toolbox Partial differential equations (PDE) are used in two mesh dimensions of triangular elements [17].

In summary, the solution of Equation (1) will go through:

In triple ordained $\left\langle A_{k}, B_{k}, \Omega_{k}\right\rangle$ it can be represented by $\Gamma_{k}$ the set of degree of freedom (nodes) in the Equation (9) it is an base of an space that genera the function $J_{k}$, see the Equation (9) it is normally a constitutive re- 
lation, and the demine $\Omega_{k}$ the geometrical space.

- Attaining the reference configuration of Equation (1).

- Solving the reference configuration representation over ${ }^{0} \Omega$ thus obtaining current and conductivity density measurements.

- Obtaining conductivity tensors and current density from the reference configuration.

- Proposing the functional of the constitutive equation which might be formulated by the Galerkin technique using the minimal residual method.

- Solving using a triangular mesh seed.

In this way the proposed technique is oriented to see the potential distribution in the $\Omega_{k}$ the geometrical space, the principal restriction in the design procedures of the standard IEEE-80 [1].

\section{Practical Example}

In the Table 1 its resume the parameter for one grid that is showed in the Figure 1.

The Table 1, $\rho$ and $\rho_{S}$ are the resistivity of the soil and surface respectively, $h_{S}$ and $h$ is the deep of the grid in the soil and electrodes longitude, $I_{0}$ is the fault current of the system, $t_{C}$ is the time protection action, and finally the $L_{1}, L_{2}$ and $L_{V}$ are the dimension of the grid. Figure 1 shows the configuration of the mesh of the grounding system implemented in the software ETAP [18]. An example, it can be seen in [19].

The graphs for step and touch voltage are given in Figure 2.

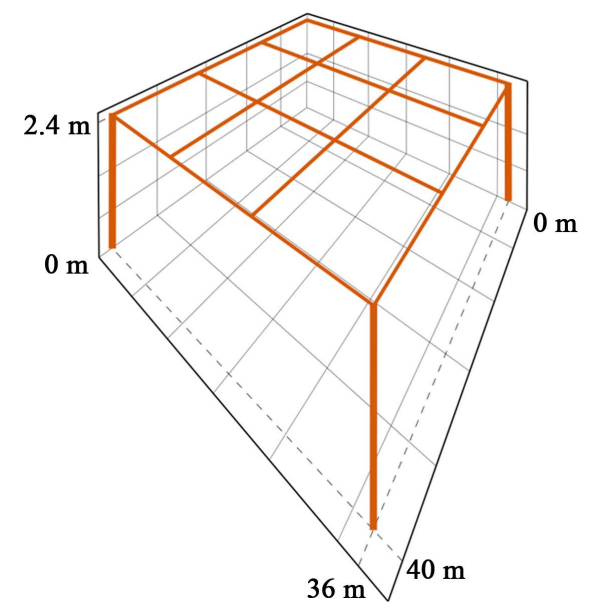

Figure 1. Geometrical distribution.

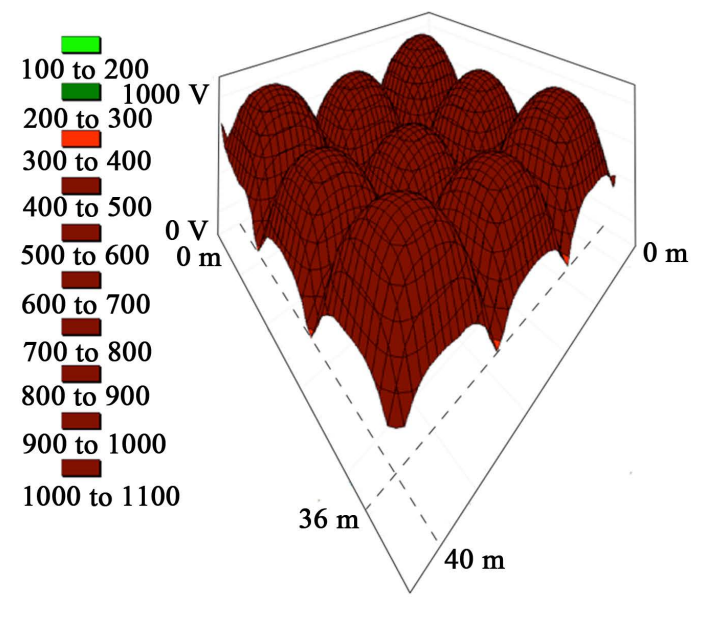

Touch voltage

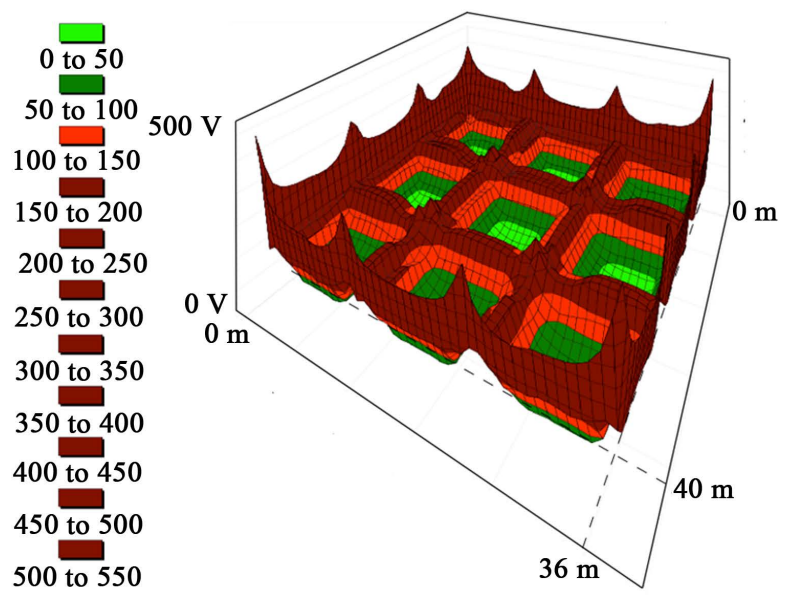

Step voltage

Figure 2. Touch and step voltages. 
Table 1. Physical parameters.

\begin{tabular}{cccccccccc}
\hline Parameter & Value & Parameter & Value & Parameter & Value & Parameter & Value & Parameter & Value \\
\hline$\rho_{S}$ & $5500 \Omega \cdot \mathrm{m}$ & $t_{C}$ & $0.5 \mathrm{~s}$ & $h$ & $0.5 \mathrm{~m}$ & $L_{2}$ & $36 \mathrm{~m}$ & $I_{0}$ & $10,000 \mathrm{~A}$ \\
$\rho$ & $459.2 \Omega \cdot \mathrm{m}$ & $L_{1}$ & $40 \mathrm{~m}$ & $h_{S}$ & $0.2 \mathrm{~m}$ & $L_{V}$ & $2.4 \mathrm{~m}$ & Grid & $13.33 \times 12 \mathrm{~m}^{2}$ \\
\hline
\end{tabular}

The program provides the information below after analyzing the ground resistance information taken from the measurements of the electrical plant.

The solution using finite element analysis, for an element with three nodes, in a distribution matrix whit 320 elements and one grid from 1200 nodes.

From the finite element solution, it is possible obtained the parameter listed below it is showed in the Figure 2:

Threshold levels of touch potential:

Elevation of ground potential: 4837 volts;

Maximum step voltage: 7650.93 volts.

\section{Comparison of Results}

The results obtained in ETAP are compared with the method discussed in the previous section this technique was developed using MATLAB [17], the step and touch voltages are given in Figure 3.

The Table 2 shows the comparison of results of the ground mesh resistance.

Table 2 shows that the results obtained are very similar which leads to the conclusion that the methods are suitable for the design and analysis of ground meshes.

This solution is so important due the necessary algorithm implementation in the optimization procedures.

\section{Dynamic 0ptimization of IEEE-80 Procedure}

The block diagram of Figure 4 illustrates the sequences of steps to design the ground grid procedure from IEEE80-2013 [1]. The parameters shown in the block diagram are identified in the index presented in Table 12 of that reference [1]. The principal parameter for that block diagram is GPR, $E_{\text {step }}$ and $E_{\text {touch }}$ Step 7, 9 and 10 respectively. We changed the step 8 it was introduced the Finite Element Method to calculate the $E_{\text {mesh }}$ and $E_{\text {touch }}$. They are the restriction of the dynamic programming approach. They can be obtained using the method described in the previous section.

As it is showed for the Figure 3, the tradition for the use of the design of power systems grid is the Dynamic programing that is so close to the dynamic optimization. The tradition by Richard Bellman's method [20]. It is to model and interpolated the behavior of model under assumption of forward looking optimizing behavior. Dynamic optimization deals with the problem of obtaining a sequence of optimal choices under given dynamic constraints. Dynamic programing is the most commonly used technique this part of the paper we are working to show a computational implementation given its recursive structure it is make in the step 11, that is an important modification to the diagram block in IEEE-80 it is showed in the Figure 3.

\section{Description of the Optimization Problem}

The grounding grid is composed for linear conductors. Each conductor is subdivided in small linear segments. We can obtain the current density in each segment using the complex images method [3]. Figure 5 shows the grounding grid.

The objective function is described by.

$$
\min \left[\sum_{x, y, k, i, j} L_{x}\left(C L_{x}\right)+L_{y}\left(C L_{y}\right)+N e_{k}\left(C N e_{k}\right)+h_{i}+C O N_{j}\right]
$$

Constrains:

$$
\begin{aligned}
& V_{\text {touch }} \leq V_{\text {touch_min }} \\
& V_{\text {step }} \leq V_{\text {step_min }} \\
& G P R \leq G P R_{\min } \\
& A \leq A_{\max }
\end{aligned}
$$




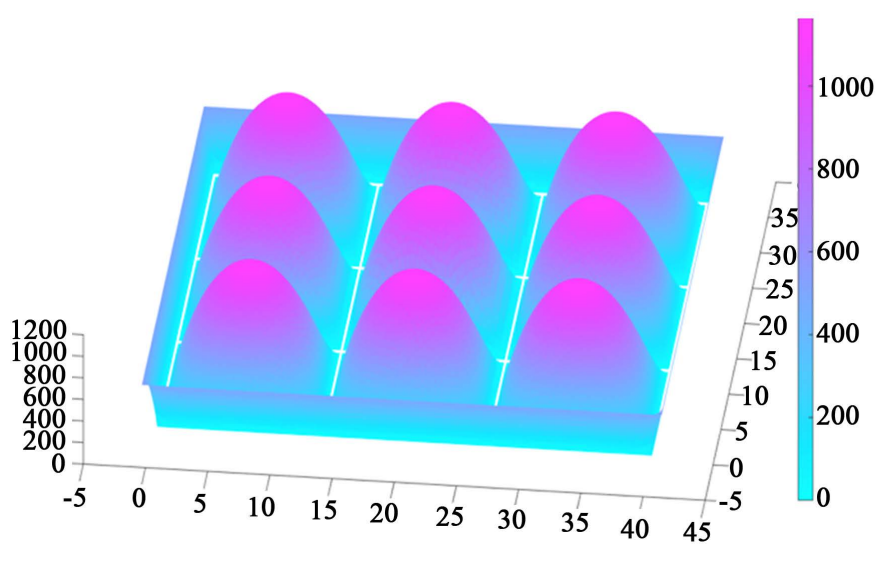

Touch voltage

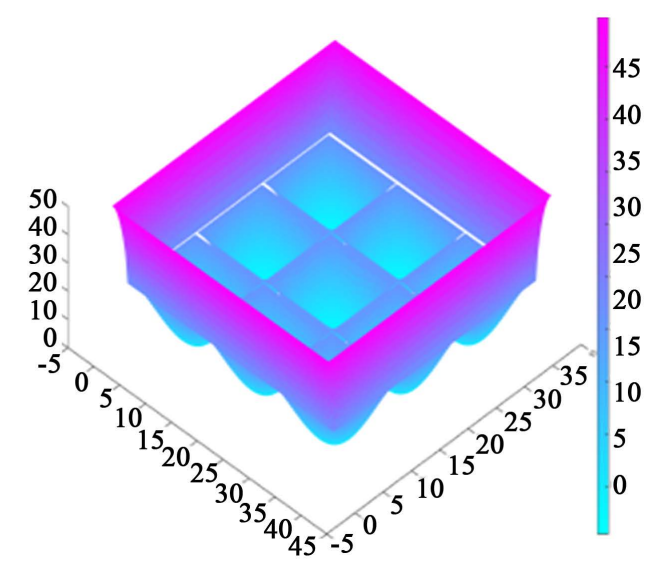

Step voltage

Figure 3. Touch and step voltages.

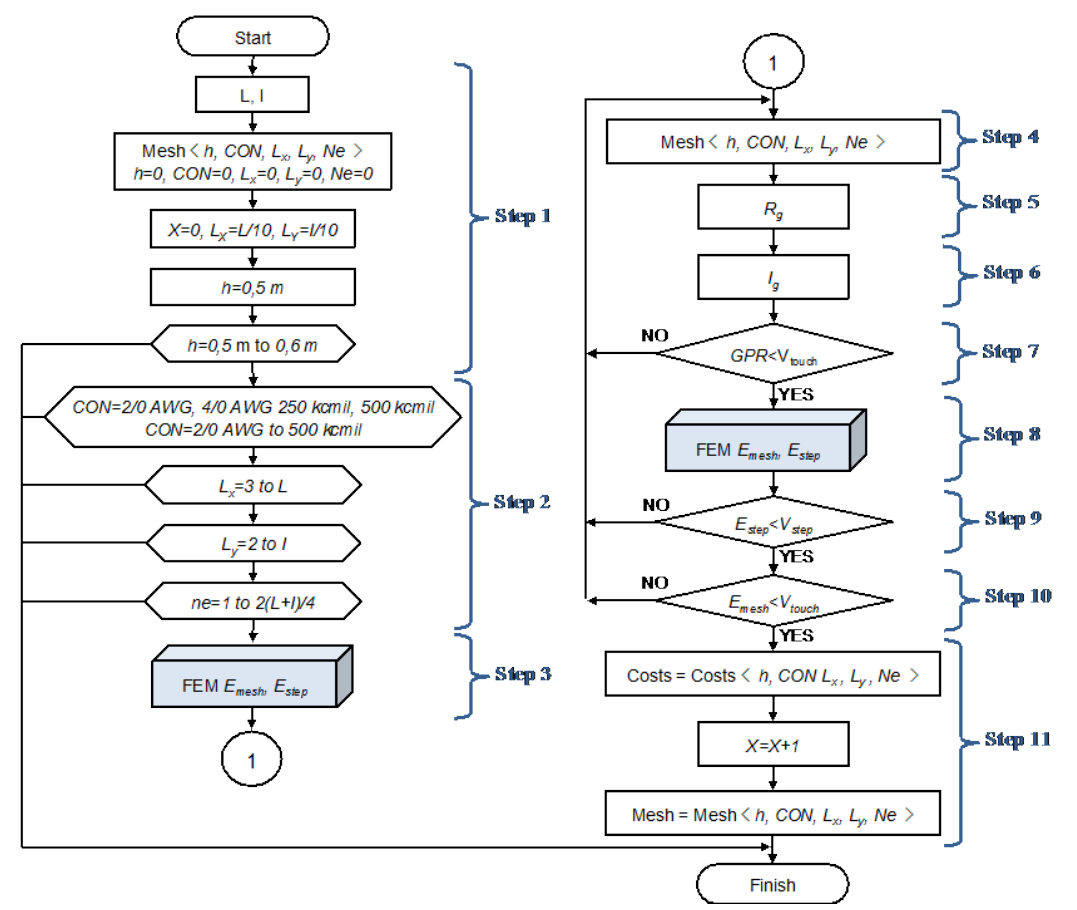

Figure 4. Dynamic programing procedure.

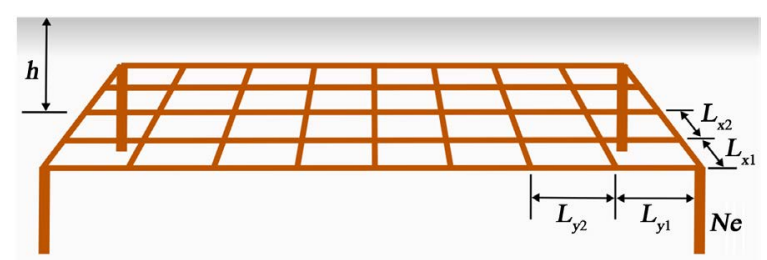

Figure 5. Components of the optimization process.

Table 2. Results of the mesh resistance. 
where:

ne: number of electrodes;

$\mathrm{CNe}$ : is the total cost of electrodes including the installation;

$L_{x}$ : number of horizontal electrodes in the direction $X$,

$C L_{X}$ : is the total cost of the electrodes including the installation;

$L_{y}$ : number of horizontal electrodes in the direction $Y$;

$C L_{y}$ : is the total cost of the electrodes including the installation;

$h$ : is the deep respect to the surface of the grid;

$C O N$ : is the cost of the conductor diameter;

A: area.

The Table 3 shows the parameter of the Equation (12).

To solve the problem, the dynamic programming approach uses a recursive method that works backwards. For the Equation (10) the method work as follows:

- Compute the cost $J$ of each component in the each grid configuration (in the last stage).

- Compute the cost of each feasible optimal sequence of component for the each node which implies the optimal sequence to the construction of the grid if optimal sequence problem.

- Its solved is optimal it ask to the constriction step 9, 10 and 12 of the flow, the Figure 4.

It is very similar to the economic application in which a structured proposed grid with the voltage as a state variable is evolves troughs time. This system can be manipulated by means of a set or blocks of construction part of the grid (electrodes and cables) in order to minimize the cost function (10).

Remember that the dynamic programming approached works by solving the problem backward in time, determining the optimal grid, it is transform the original (10) problem and an subsequence of sub-problems. It is a crucial notion of the cost-to-go, which is the cost along minimum-cost path from a given grid stated.

The general approach:

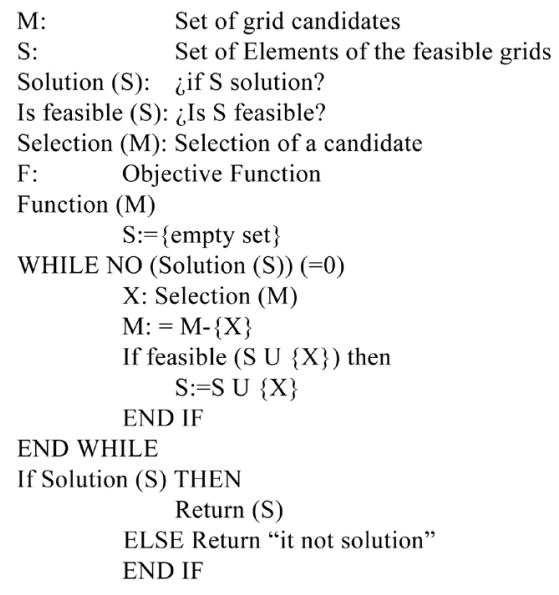

Figure 6 shows the configuration of the grounding system optimal. The Table 4 is the result from application of this algorithm to the optimal design problem in an $115 \mathrm{kV}$ substation and therefore the grounding system optimal is the number 21 (Grid21) of the table.

Table 3. Parameters used in the Equation (12).

\begin{tabular}{cc}
\hline Element & Cost \\
Ne & $\$ 35.00$ \\
CON 4/0 AWG & $\$ 12.50$ \\
CON 250 kcmil & $\$ 45.00$ \\
Installation cost (m $\left.{ }^{2}\right)$ & $\$ 7.00$ \\
Installation cost (electrode) & $\$ 8.00$ \\
\hline
\end{tabular}




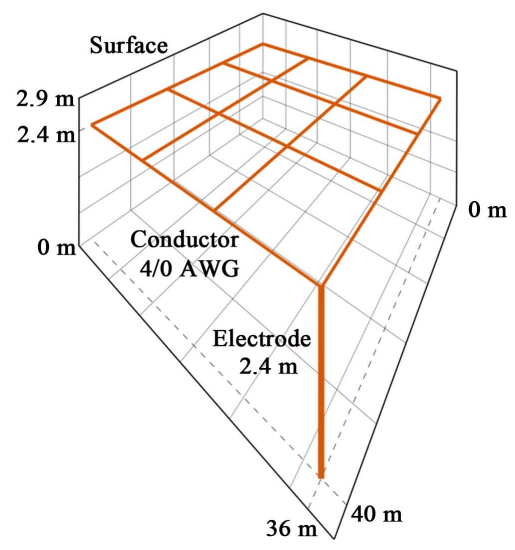

Figure 6. Optimal ground system.

Table 4. Results of the optimization process.

\begin{tabular}{|c|c|c|c|c|c|c|c|c|c|c|c|c|}
\hline \multirow{2}{*}{ GRID } & \multirow{2}{*}{$h$} & \multirow{2}{*}{ CON } & \multirow{2}{*}{$\mathrm{Ne}$} & \multirow{2}{*}{$R_{g}$} & \multirow{2}{*}{$G P R$} & \multicolumn{2}{|c|}{$E_{\text {touch }}$} & \multicolumn{2}{|c|}{$E_{\text {step }}$} & \multicolumn{2}{|c|}{ Cost } & \multirow{2}{*}{$\begin{array}{c}\text { \$Total } \\
\text { Cost }\end{array}$} \\
\hline & & & & & & Tolerable & Calculated & Tolerable & Calculated & \$Electrode & \$Conductor & \\
\hline Grid1 & 0.5 & $4 / 0$ & 0 & 0.426 & 4825.7 & 1231.0 & 2685.9 & 4373.7 & 762.6 & $\$ 0$ & $\$ 11.980$ & $\$ 11.980$ \\
\hline Grid2 & 0.5 & $4 / 0$ & 0 & 0.392 & 4443.2 & 1231.0 & 1746.5 & 4373.7 & 615.5 & $\$ 0$ & $\$ 12.930$ & $\$ 12.930$ \\
\hline Grid3 & 0.5 & $4 / 0$ & 0 & 0.375 & 4240.8 & 1231.0 & 1326.2 & 4373.7 & 554.8 & $\$ 0$ & $\$ 13.880$ & $\$ 13.880$ \\
\hline Grid4 & 0.5 & 250 & 0 & 0.373 & 4228.4 & 1231.0 & 1310.9 & 4373.7 & 554.8 & $\$ 0$ & $\$ 23.760$ & $\$ 23.760$ \\
\hline Grid5 & 0.5 & 250 & 0 & 0.389 & 4407.3 & 1231.0 & 1698.9 & 4373.7 & 524.3 & $\$ 0$ & $\$ 20.340$ & $\$ 20.340$ \\
\hline Grid6 & 0.5 & 250 & 0 & 0.419 & 4748.1 & 1231.0 & 2614.7 & 4373.7 & 641.2 & $\$ 0$ & $\$ 16.920$ & $\$ 16.920$ \\
\hline Grid7 & 0.6 & $4 / 0$ & 0 & 0.422 & 4772.9 & 1231.0 & 2638.0 & 4373.7 & 641.2 & $\$ 0$ & $\$ 11.980$ & $\$ 11.980$ \\
\hline Grid8 & 0.6 & $4 / 0$ & 0 & 0.391 & 4423.8 & 1231.0 & 1716.9 & 4373.7 & 524.3 & $\$ 0$ & $\$ 12.930$ & $\$ 12.930$ \\
\hline Grid9 & 0.6 & $4 / 0$ & 0 & 0.372 & 4214.4 & 1231.0 & 1298.3 & 4373.7 & 474.7 & $\$ 0$ & $\$ 13.880$ & $\$ 13.880$ \\
\hline Grid10 & 0.6 & $4 / 0$ & 0 & 0.419 & 4748.1 & 1231.0 & 2614.7 & 4373.7 & 641.2 & $\$ 0$ & $\$ 11.980$ & $\$ 11.980$ \\
\hline Grid11 & 0.6 & $4 / 0$ & 0 & 0.389 & 4407.3 & 1231.0 & 1698.9 & 4373.7 & 524.3 & $\$ 0$ & $\$ 12.930$ & $\$ 12.930$ \\
\hline Grid12 & 0.6 & 250 & 0 & 0.371 & 4202.0 & 1231.0 & 1283.0 & 4373.7 & 474.7 & $\$ 0$ & $\$ 23.760$ & $\$ 23.760$ \\
\hline Grid13 & 0.5 & $4 / 0$ & 1 & 0.425 & 4806.4 & 1231.0 & 2592.1 & 4373.7 & 749.2 & $\$ 43$ & $\$ 11.980$ & $\$ 12.023$ \\
\hline Grid14 & 0.5 & $4 / 0$ & 1 & 0.393 & 4450.4 & 1231.0 & 1641.3 & 4373.7 & 610.8 & $\$ 43$ & $\$ 12.930$ & $\$ 12.973$ \\
\hline Grid15 & 0.5 & $4 / 0$ & 1 & 0.374 & 4235.9 & 1231.0 & 1213.0 & 4373.7 & 549.8 & $\$ 43$ & $\$ 13.880$ & $\$ 13.923$ \\
\hline Grid16 & 0.5 & 250 & 1 & 0.422 & 4782.3 & 1231.0 & 2569.4 & 4373.7 & 749.2 & $\$ 43$ & $\$ 16.920$ & $\$ 16.963$ \\
\hline Grid17 & 0.5 & 250 & 1 & 0.392 & 4434.2 & 1231.0 & 1623.7 & 4373.7 & 610.8 & $\$ 43$ & $\$ 20.340$ & $\$ 20.383$ \\
\hline Grid18 & 0.5 & 250 & 1 & 0.373 & 4223.7 & 1231.0 & 1197.9 & 4373.7 & 549.8 & $\$ 43$ & $\$ 23.760$ & $\$ 23.803$ \\
\hline Grid19 & 0.6 & $4 / 0$ & 1 & 0.420 & 4755.0 & 1231.0 & 2546.2 & 4373.7 & 629.9 & $\$ 43$ & $\$ 11.980$ & $\$ 12.023$ \\
\hline Grid20 & 0.6 & $4 / 0$ & 1 & 0.390 & 4415.8 & 1231.0 & 1609.6 & 4373.7 & 518.1 & $\$ 43$ & $\$ 12.930$ & $\$ 12.973$ \\
\hline Grid21 & 0.6 & $4 / 0$ & 1 & 0.372 & 4209.9 & 1231.0 & 1188.5 & 4373.7 & 470.5 & $\$ 43$ & $\$ 13.880$ & $\$ 13.923$ \\
\hline Grid22 & 0.6 & 250 & 1 & 0.418 & 4730.9 & 1231.0 & 2523.5 & 4373.7 & 629.9 & $\$ 43$ & $\$ 16.920$ & $\$ 16.963$ \\
\hline Grid23 & 0.6 & 250 & 1 & 0.389 & 4399.6 & 1231.0 & 1591.9 & 4373.7 & 518.1 & $\$ 43$ & $\$ 20.340$ & $\$ 20.383$ \\
\hline
\end{tabular}




\section{Continued}

\begin{tabular}{|c|c|c|c|c|c|c|c|c|c|c|c|c|}
\hline Grid24 & 0.6 & 250 & 1 & 0.371 & 4197.7 & 1231.0 & 1173.3 & 4373.7 & 470.5 & $\$ 43$ & $\$ 23.760$ & $\$ 23.803$ \\
\hline Grid25 & 0.5 & $4 / 0$ & 2 & 0.374 & 4230.9 & 1231.0 & 1198.0 & 4373.7 & 545.0 & $\$ 86$ & $\$ 13.880$ & $\$ 13.966$ \\
\hline Grid26 & 0.5 & $4 / 0$ & 2 & 0.392 & 4441.5 & 1231.0 & 1614.5 & 4373.7 & 603.7 & $\$ 86$ & $\$ 12.930$ & $\$ 13.016$ \\
\hline Grid27 & 0.5 & $4 / 0$ & 2 & 0.422 & 4776.7 & 1231.0 & 2495.7 & 4373.7 & 729.4 & $\$ 86$ & $\$ 11.980$ & $\$ 12.066$ \\
\hline Grid28 & 0.5 & 250 & 2 & 0.421 & 4763.2 & 1231.0 & 2507.5 & 4373.7 & 736.2 & $\$ 86$ & $\$ 16.920$ & $\$ 17.006$ \\
\hline Grid29 & 0.5 & 250 & 2 & 0.391 & 4425.5 & 1231.0 & 1597.1 & 4373.7 & 603.7 & $\$ 86$ & $\$ 20.340$ & $\$ 20.426$ \\
\hline Grid30 & 0.5 & 250 & 2 & 0.373 & 4218.8 & 1231.0 & 1183.1 & 4373.7 & 545.0 & $\$ 86$ & $\$ 23.760$ & $\$ 23.846$ \\
\hline Grid31 & 0.6 & $4 / 0$ & 2 & 0.418 & 4736.7 & 1231.0 & 2484.9 & 4373.7 & 619.0 & $\$ 86$ & $\$ 11.980$ & $\$ 12.066$ \\
\hline Grid32 & 0.6 & $4 / 0$ & 2 & 0.389 & 4407.6 & 1231.0 & 1583.3 & 4373.7 & 512.1 & $\$ 86$ & $\$ 12.930$ & $\$ 13.016$ \\
\hline Grid33 & 0.6 & $4 / 0$ & 2 & 0.371 & 4205.2 & 1231.0 & 1173.8 & 4373.7 & 466.4 & $\$ 86$ & $\$ 13.880$ & $\$ 13.966$ \\
\hline Grid34 & 0.6 & 250 & 2 & 0.416 & 4713.2 & 1231.0 & 2462.7 & 4373.7 & 619.0 & $\$ 86$ & $\$ 16.920$ & $\$ 17.006$ \\
\hline Grid35 & 0.6 & 250 & 2 & 0.388 & 4391.6 & 1231.0 & 1565.9 & 4373.7 & 512.1 & $\$ 86$ & $\$ 20.340$ & $\$ 20.426$ \\
\hline Grid36 & 0.6 & 250 & 2 & 0.370 & 4193.1 & 1231.0 & 1150.8 & 4373.7 & 464.2 & $\$ 86$ & $\$ 23.760$ & $\$ 23.846$ \\
\hline Grid37 & 0.5 & $4 / 0$ & 3 & 0.421 & 4766.9 & 1231.0 & 2470.2 & 4373.7 & 723.8 & $\$ 129$ & $\$ 11.980$ & $\$ 12.109$ \\
\hline Grid38 & 0.5 & $4 / 0$ & 3 & 0.391 & 4432.4 & 1231.0 & 1588.6 & 4373.7 & 596.7 & $\$ 129$ & $\$ 12.930$ & $\$ 13.059$ \\
\hline Grid39 & 0.5 & $4 / 0$ & 3 & 0.373 & 4225.7 & 1231.0 & 1183.4 & 4373.7 & 540.3 & $\$ 129$ & $\$ 13.880$ & $\$ 14.009$ \\
\hline Grid40 & 0.5 & 250 & 3 & 0.419 & 4743.9 & 1231.0 & 2448.5 & 4373.7 & 723.7 & $\$ 129$ & $\$ 16.920$ & $\$ 17.049$ \\
\hline Grid41 & 0.5 & 250 & 3 & 0.401 & 4541.8 & 1231.0 & 1585.0 & 4373.7 & 611.2 & $\$ 129$ & $\$ 20.340$ & $\$ 20.469$ \\
\hline Grid42 & 0.5 & 250 & 3 & 0.372 & 4213.8 & 1231.0 & 1168.7 & 4373.7 & 540.3 & $\$ 129$ & $\$ 23.760$ & $\$ 23.889$ \\
\hline Grid43 & 0.6 & $4 / 0$ & 3 & 0.417 & 4718.2 & 1231.0 & 2426.4 & 4373.7 & 608.5 & $\$ 129$ & $\$ 11.980$ & $\$ 12.109$ \\
\hline Grid44 & 0.6 & $4 / 0$ & 3 & 0.389 & 4399.2 & 1231.0 & 1557.9 & 4373.7 & 506.2 & $\$ 129$ & $\$ 12.930$ & $\$ 13.059$ \\
\hline Grid45 & 0.6 & $4 / 0$ & 3 & 0.371 & 4200.4 & 1231.0 & 1159.5 & 4373.7 & 462.3 & $\$ 129$ & $\$ 13.880$ & $\$ 14.009$ \\
\hline Grid46 & 0.6 & 250 & 3 & 0.415 & 4695.3 & 1231.0 & 2404.7 & 4373.7 & 608.5 & $\$ 129$ & $\$ 16.920$ & $\$ 17.049$ \\
\hline Grid47 & 0.6 & 250 & 3 & 0.387 & 4383.5 & 1231.0 & 1540.7 & 4373.7 & 506.2 & $\$ 129$ & $\$ 20.340$ & $\$ 20.469$ \\
\hline Grid48 & 0.6 & 250 & 3 & 0.4 & 4188.5 & 1231.0 & 1144.7 & 4373.7 & 462.3 & $\$ 129$ & $\$ 23.760$ & $\$ 23.889$ \\
\hline
\end{tabular}

\section{Conclusion}

In the present paper we discussed the application of the finite element method and dynamic programing to the design of the ground power systems (electrodes and mesh ground). Considering that these procedures are normally used in other areas, it is very important to introduce physical knowledge as much as possible, in order to "guide" the solution in the case of IEEE 80-2013 standard application in the particular case of Electrical Engineering work.

\section{References}

[1] ANSI/IEEE Standard 80-2013 Guide for Safety in AC Substations Grounding.

[2] Practical Applications of ANSI/IEEE Standard 80-1986-IEEE Guide for Safety. Garret, D.L Org., 86 EhO253-S-PWR.

[3] Casas Ospina, F. (2010) Grounding—Safety in Power Systems (Spanish). INCONTEC, 187p,

[4] Zienkiewicz, O.C., Taylor, R.L. and Zhu, J.Z. (2005) The Finite Element Method. 6th Edition, Elsevier, Barcelona.

[5] Reddy, J.N. (2005) An Introduction to the Finite Element Method. McGraw-Hill, New York.

[6] Cano Plata, E.A. and Ramirez Castaño, J.S. (2010) Systems Grid Ground: Design with IEEE-80 and Evaluated with 
FEM (Spanish). National University of Colombia, Manizales.

[7] Simo, J.C. (1988) A Framework for Finite Strain Elasto-Plasticity Based on Maximum Plastic Dissipation and the Multiplicative Decomposition, Part I: Continuum Formulation. Computer Methods in Applied Mechanics and Engineering, 66, 199-219. http://dx.doi.org/10.1016/0045-7825(88)90076-X

[8] Simo, J.C. (1988) A Framework for Finite Strain Elasto-Plasticity Based on Maximum Plastic Dissipation and the Multiplicative Decomposition, Part II: Computational Aspects. Computer Methods in Applied Mechanics and Engineering, 68, 199-219. http://dx.doi.org/10.1016/0045-7825(88)90076-X

[9] Simo, J.C. and Marsden, J.E. (1984) On the Rotated Stress Tensor and the Material Version of the Doyle-Ericksen Formula. Archive for Rational Mechanics and Analysis, 86, 213-231. http://dx.doi.org/10.1007/BF00281556

[10] Michiel, H. (2001) Differentiable Manifold. Encyclopedia of Mathematics. Springer, Berlin.

[11] Monk, P. (2003) Finite Element Methods for Maxwell’s Equations, Numerical Mathematics and Scientific Computation. Clarendon Press, Oxford, 450.

[12] Anand, L. (1979) On Hencky’s Approximate Strain-Energy Function for Moderate Deformation. Journal of Applied Mechanics, 46, 78-82. http://dx.doi.org/10.1115/1.3424532

[13] Rolph III, W.D. and Bathe, K.J. (1984) On a Large Strain Finite Element Formulation for Elasto-Plastic Analysis. In: William, K.J., Ed., Constitutive Equations: Macro and Computational Aspects, Winter Annual Meeting, ASME, New York, 131-147.

[14] Weber, G. and Anand, L. (1990) Finite Deformation Constitutive Equation and a Time Integration Procedure for Isotropic, Hyperelastic-Viscoplastic Solids. Computer Methods in Applied Mechanics and Engineering, 79, $173-202$. (1990). http://dx.doi.org/10.1016/0045-7825(90)90131-5

[15] Eterovic, A.L. and Bathe, K.J. (1990) A Hyperelastic Based Large Strain Elasto-Plastic Constitutive Formulation with Combined Isotropic Kinematic Hardening Using the Logarithmic Stress and Strain Measures. International Journal for Numerical Methods in Engineering, 30, 1099-1114. http://dx.doi.org/10.1002/nme.1620300602

[16] Dvorki, E. and Goldschmit, M. (2002) Finite Element Method, Graduate Course. Universidad de Buenos Aires, Buenos Aires.

[17] Matlab Partial Differential Equation (PDE) Toolbox, MATLAB 7.0, 2008.

[18] ETAP 114C Power System Engineering, Operation Software Technology, 2014—Grounding Module.

[19] Soto Marin, O.J. (2015) Failure Analysis of Distribution Transformers in the East Zone of Caldas. National University of Colombia, Manizales.

[20] Bellman, R.E. (1957) Dynamic Programming. Princeton University Press, Princeton. 